\title{
Diagnostic yield for endoscopy in patients with altered bowel habits
}

\begin{abstract}
Background: Alterations in bowel habits is one of leading complaints among patients seen in the gastroenterology office. The increased volume of patient with complaints of chronic diarrhea, constipation or altered bowel habits continues to raise the question of the appropriateness and role for endoscopy as part of the diagnostic workup regarding these increasingly prevalent complaints. There is currently no clear evidence as to when endoscopy should be performed in evaluation of altered bowel habits. Many of the causative pathological processes resulting in altered bowel habits would not be detected on endoscopy or pathology and therefore the suspected diagnostic yield would be low. We suspect that the risk and cost associated with endoscopy relative to the low diagnostic yield should cause clinicians to focus efforts on other diagnostic measures prior to recommending or performing endoscopy for chronic diarrhea, chronic constipation, or altered bowel habits.
\end{abstract}

Objectives: The goal of this study is to confirm suspicion that the diagnostic yield for anatomic and histological etiologies on endoscopy in patients with altered bowel habits in a diverse community based hospital setting is significantly low. This should prompt patients to have further work up and evaluation through other means. Therefore, endoscopy without other indications should not be part of the routine or initial work up and selectively applied to patients only after negative initial workups have been performed.

Methods: We performed a retrospective study of patients with complaints of $\mathrm{ABH}$ (defined as altered bowel habits for greater than 6 weeks, chronic diarrhea for greater than 6 weeks, or chronic constipation greater than 6 weeks) from January 1, 2005 through October 9, 2015 at South Oakland Gastroenterology Associates (SOGA) in Farmington Hills, Michigan who underwent open access endoscopy (some patients had been referred and were seen for the first time by the gastroenterologist on the day of their procedure). Data was analyzed from 297 patients and we determined their diagnostic yield based off the anatomic and histological findings that were felt could adequately explain the patient's symptoms after performing endoscopy.

Results: A total of 300 patients were originally identified but 3 were removed from further analysis due to poor preparations. The remaining 297 patient were identified included for full analysis. 108 were evaluated for complaint of diarrhea, 46 for constipation, and 143 for altered bowel habits. 208 patients underwent colonoscopy alone evaluation. 83 underwent combined EGD and colonoscopy evaluation. 6 underwent EGD alone evaluation. There were 103 patients with abnormal histology and 32 (10.77\% of total patient population) patients that had histological diagnosis (IBD, Ischemic colitis, microscopic colitis, infectious colitis, cancer, or celiac disease) that provided a clear diagnosis to explain the patient's complaints. A much higher yield involving the anatomical findings of polyps, inflammatory changes, mucosal injury, mass, or stricture was noted in $152(51.18 \%)$ of the patients. However, it
Volume 6 Issue 2 - 2017

\author{
Chistopher Wood,' Ward C Zeno, ${ }^{2}$ Robert \\ Jarski, ${ }^{3}$ Mariquit Sendelbach, ${ }^{4}$ Robert \\ Bischoff, ${ }^{5}$ Mark Raphael ${ }^{5}$ \\ 'Department of Gastroenterology and Hepatology, Beaumont \\ Health - Farmington Hills, USA \\ ${ }^{2}$ South Oakland Gastroenterology Associates, Farmington Hills, \\ USA \\ ${ }^{3}$ Garden City Hospital, USA \\ ${ }^{4}$ Detroit Medical Center Huron Valley- Sinai Hospital, USA \\ ${ }^{5}$ Lakes Surgery Center,West Bloomfield, USA
}

Correspondence: Chistopher Wood, Beaumont Health Farmington Hills, Farmington Hills, Michigan, USA, Email doctoc.do@gmail.com

Received: December 01, 2016 | Published: February 16, 2017

should be noted that many of the anatomical diagnosis did not clearly explain the patient's complaint. Of the 152 anatomically significant findings 101 were polyps with $54(53.5 \%)$ being adenomatous. When including only the anatomical finding that were suggestive of being responsible for the patients symptoms only 43 (including polyps suspicious for diarrhea, inflammatory changes, and one recorded stricture) were included. The diagnostic yield of endoscopy for clearly identifying diagnosis responsible or suggestive for chronic altered bowel habits, chronic diarrhea, or chronic constipation was very low for the patients in our small community based study. The data recorded demonstrated $10.77 \%$ for histological confirmed diagnosis and $14.48 \%$ anatomically suggestive diagnosis that would explain the patient's complaints.

Conclusion: This study showed that endoscopy is unlikely to uncover a diagnosis to explain altered bowel habits in the general population. Given the low diagnostic yield of endoscopic evaluation for altered bowel habits, we believe that an initial noninvasive workup is reasonable to identify a likely diagnosis prior to any endoscopic evaluation. In patients that have confirmed diagnosis by histology, we did note that a significant percentage of these patients had normal endoscopic exams. This should encourage that, when evaluating patients with altered bowel habits, biopsies should be taken even if gross appearance is normal.

\section{Introduction}

There are many proper indications to perform endoscopy, including gastrointestinal bleeding, weight loss, and colon cancer screening. Evidence is clear for these indications, but there has yet to be any clear evidence as to when, and if, endoscopy should be performed for chronic ABH. Endoscopy is not a risk-free procedure, nor is it non-invasive. Complications such as bleeding, perforation, infection, or complications from anesthesia can occur. The proper precautions should be taken prior to having this procedure done to ensure that there no unnecessary risk undertaken by the patient. $\mathrm{ABH}$ are an increasing issue to the U.S. population, and with that, there needs to more evidence to support its utility in the diagnostic workup and management of chronic ABH. Laboratory data, imaging, and stool studies are common initial work up, both in the primary care setting and the gastroenterology setting. But when the initial testing is negative, the question still remains as to when more invasive testing should be done.

Inflammatory bowel disease, irritable bowel syndromes, prior gastrointestinal surgeries, and specific dietary intolerances are just a few of the common causes of ABH. Previous studies have evaluated the yield of endoscopy in patients complaining of $\mathrm{ABH}$ without some of these pre-existing conditions, but not all. Such studies evaluated patients with prior gastrointestinal surgery or abdominal pain as the 
primary indications for endoscopy that was used to evaluate $\mathrm{ABH}$. Also, past studies have investigated the need for colonoscopy or esophagogastroduodenoscopy (EGD) for diarrhea, constipation, or abdominal pain. These studies however did not look at pathology diagnosis as part of their endpoints. This effectively limits several possible causes of diarrhea, constipation, or other $\mathrm{ABH}$. In addition, these studies did not specifically define diarrhea and constipation as either acute or chronic. To address these issues, we conducted a retrospective study using SOGA's electronic medical records. The yield of endoscopy was investigated for patients with complaints of chronic diarrhea, chronic constipation, or $\mathrm{ABH}$ and no pre-existing medical conditions, listed in the exclusion criteria, that could contribute to their symptoms.

\section{Methods}

\section{Setting}

This retrospective study was conducted among patients of South Oakland Gastroenterology Associates (SOGA) practice, located in Farmington Hills, Michigan. Other office branches and endoscopy centers that were used are located in Commerce Township, MI, Garden City, MI, Livonia, MI, and West Bloomfield, MI, therefore providing care to a substantial number of patients with racial and social diversity in Southeastern Michigan.

\section{Study design and patients}

As previously noted, this study was performed to evaluate the diagnostic yield of endoscopy in patient with chronic diarrhea, chronic constipation, or altered bowel habits. This study is different than prior studies in that it collectively looks at patients with altered bowel habits, those with defined chronic diarrhea and constipation. Diarrhea we defined 'chronic' as at least 6 weeks of symptoms. Additionally, pathology was taken into account as part of the diagnostic yield of conditions that have been individually noted to be responsible for the patients' symptoms, albeit rare. This study was reviewed and approved by the Beaumont Hospital Institutional Review Board prior to the beginning of any data collection or collective review.

It was conducted as a retrospective chart review using electronic medical record (EMR) charts from the office of the SOGA group. The medical software utilized by SOGA is Aprima ${ }^{\circledR}$. The initial patient population was provided by reviewing EMR endoscopy scheduling records for patients that had undergone endoscopy evaluations. The patients identified by EMR scheduling were then searched and the charts reviewed to identify patients that met the study inclusion criteria. All charts were reviewed by trained physicians to identify appropriate patients for the study as outlined by the inclusion and exclusion criteria. All patients with insufficient records to adequately verify they did not have any of the exclusion criteria were excluded from the study to avoid adding potential patients that did in fact meet exclusion criteria that was unknown at the time of chart review. For example, patients were excluded if they had prior bowel surgery. If surgical history was listed as unknown these patients were also excluded as the chart could not properly identify them as NOT having had any prior bowel surgery. The same process of exclusion was applied for all exclusion criteria.

\section{Inclusion criteria:}

i. Patient ages 16-89 years old.

ii. Must have at least 6 week history of diarrhea, constipation, or altered bowel habits.

\section{Exclusion criteria:}

i. Patients $<16$.

ii. Patients $>89$.

iii. Patients with acute diarrhea lasting less than 6 weeks.

iv. Patients with acute constipation lasting less than 6 weeks.

v. Patients with previous gastrointestinal surgery such as bowel resection or bypass.

vi. Patients with a known medical condition or medications that could be contributing to the diarrhea and/or constipation. (This would include inflammatory bowel disease, irritable bowel syndrome, Celiac disease, microscopic colitis, or known dietary intolerances such as lactose intolerance.

Endoscopy procedure reports were reviewed for 3219 patients. A total of 5118 individual endoscopy reports were reviewed to identify patients between the ages of 16 and 89 years old whom had endoscopy performed with a chief complaint of $\mathrm{ABH}$, chronic diarrhea, or chronic constipation from January 1, 2005 to October 9, 2015. SOGA's electronic medical records were used to identify these patients and the patient's MRN was used as the HIPAA identifier and linking key among the list of patients. A total of 297 patients (Table 1) were identified after chart review of 3219 patients and 5118 endoscopy reports to meet inclusion criteria.

Table I Break down of patient procedures per complaint

\begin{tabular}{lllll}
\hline & Total & Diarrhea & Constipation & $\begin{array}{l}\text { Altered } \\
\text { Bowel Habit }\end{array}$ \\
\hline EGD & 6 & 3 & 0 & 3 \\
Colonoscopy & 208 & 80 & 32 & 96 \\
EGD/ & 83 & 25 & 14 & 44 \\
$\begin{array}{l}\text { Colonoscopy } \\
\text { Totals }\end{array}$ & 297 & 108 & 46 & 143 \\
\hline
\end{tabular}

The study patients' information was collected onto a Microsoft Excel spreadsheet according to a data key. The data was all placed into a secured data collection tool as outlined by the IRB. The following data was collected: Patient name and MRN (for initial identification only and later substituted for study ID numbers), attending physician name, patient age, gender, ethnicity, indication for procedure, type of procedure performed, anatomical diagnosis, and histological diagnosis.

Following data entry, every patient was assigned a patient identifier and the collected data was then securely sent for statistical analysis (Table 2).

Table 2 Patient population baseline characteristics

\begin{tabular}{lll}
\hline Gender & Count & Percent (\%): \\
\hline Male & 102 & 34.34 \\
Female & 195 & 65.66 \\
Race & & \\
White & 223 & 75.08 \\
Non-White & 74 & 24.92 \\
Age (yrs) & Mean: & Std. Deviation \\
16-86 & 52.28 & 15.63 \\
\hline
\end{tabular}




\section{Statistical analysis}

Categorical data were summarized as counts and percentages and compared by calculating the chi-square test of independence. Throughout this study a $p$-value $<0.05$ (two-tailed) was considered statistically significant. Following initial data entry using Microsoft Excel, Minitab Statistical Software (State College, PA) was used for performing the analyses.

\section{Results}

There were 297 patients included in this study to determine the diagnostic yield of endoscopy in patients with chronic altered bowel habits. Of the 297 patients, 6 underwent EGD, 208 colonoscopy and $83 \mathrm{EGD/Colonoscopy.} \mathrm{Normal} \mathrm{anatomy} \mathrm{as} \mathrm{well} \mathrm{as} \mathrm{hemorrhoids}$ and diverticulosis were grouped together as not causing the patients symptoms. All other anatomical variations were categorized as significant anatomical findings. These anatomical findings, although significant, were not all potentially responsible for the patients' symptoms. There were 152 patients that were noted to have anatomical findings other than hemorrhoids and diverticulosis. Of those 152 , only 43 (28.29\% of anatomical findings) demonstrated findings that could potentially explain the patients symptoms. $51.18 \%$ of the total patient population did have anatomical findings other than hemorrhoids and diverticulosis, but only 43 of the 152 had findings that could potentially explain the patients' symptoms. This means that the overall yield for all patients was $14.48 \%$ for an anatomical finding that could be suggestive of causing chronic alterations in bowel habits. Further evaluation of the histology revealed that 103 total patients demonstrated abnormal histology findings. However, only 32 of those patients had histology that accurately and definitively provided a diagnosis that explained the patients' alteration in bowel habits. The overall yield for positive histology was $34.68 \%$. Only $31.1 \%$ of those 103 patients were noted to have definitive diagnosis answering the question being investigated, providing an overall yield of $10.77 \%$ of total patients included.

In an effort to determine the best approach for patients, each endoscopic group (EGD, Colonoscopy, and EGD/Colonoscopy) were compared to determine the yield between groups. This comparison was done for both anatomical and histological findings. The EGD alone group was significantly smaller, with a total number of only 6 . This low number, in comparison, was too small of a sample size to accurately compare between groups, and it was therefore eliminated from the group comparison. Of the 208 patients that underwent colonoscopy evaluation alone, $93(44.71 \%)$ were identified with anatomical findings other than hemorrhoids and diverticulosis. In the EGD/Colonoscopy group, 55 (66.27\%) were noted to have anatomical findings other than hemorrhoids and diverticulosis. This yield was statistically significant with a $\mathrm{P}$-value of $\mathrm{P}=0.003$. In review of this it is felt that the combination of both EGD and Colonoscopy provided a higher combined yield of gross findings. The same process was utilized to compare the Colonoscopy group to the EGD/Colonoscopy group in reference to histological findings. A lower total yield of positive histology was noted with only 103 patients $(34.68 \%)$. The Colonoscopy group yielded $68(32.69 \%)$ with positive histology. The EGD/Colonoscopy group had $33(39.76 \%)$ with positive histology. When compared, no statistical significance was noted with a P-value of $\mathrm{P}=0.25$. It is felt that this similarity between colonoscopy alone and EGD/Colonoscopy groups was due to a decrease in incidental findings as histology was provided from targeted sampling. This further supports that as targeted tissue sampling has a more consistent yield regardless of the type of procedure performed (Table 3 ).
Table 3 Anatomical and histological diagnosis that resulted in diarrhea, constipation, or $\mathrm{ABH}$

\begin{tabular}{lll}
\hline Histology & \multicolumn{2}{l}{ Count } \\
\hline Inflammatory Bowel Disease & 12 & 4 \\
Ischemic Colitis & $\mathrm{I}$ & 0.3 \\
Microscopic Colitis & 13 & 4.4 \\
Infectious Process & 2 & 0.7 \\
Cancer & $\mathrm{I}$ & 0.3 \\
Celiac disease & 3 & $\mathrm{I}$ \\
Totals: & 32 & 10.77 \\
Anatomical & & \\
Inflammatory lesions including suspicious & 42 & 14.1 \\
polyps that could cause ABH & $\mathrm{I}$ & 0.3 \\
Strictures & 43 & 14.48 \\
Total & & \\
\hline
\end{tabular}

\section{Discussion}

The number of patients complaining of chronic $\mathrm{ABH}$, chronic diarrhea, and chronic constipation is on the rise within the U.S. Therefore, the approach to this complaint is critical, both within the primary care setting and the gastroenterology setting. In this retrospective study of 297 patients, multiple observations were made about diagnostic endoscopy for chronic $\mathrm{ABH}$. When it came to the endoscopic diagnosis, a total of 43 patients (14.48\%) were found to have a potential anatomic reason for their chronic $\mathrm{ABH}$, including inflammatory changes or a stricture. No obstructive masses were observed from the 297 patients. Mucosal inflammation and tubulovillous appearing adenomas were considered "inflammatory changes." Mucosal inflammation could be due to a multitude of etiologies, including inflammatory bowel disease, ischemic colitis, or infectious colitis. All of these could cause diarrhea, along with tubulovillous adenomas, which have also been known to cause diarrhea (though rare unless large). None of the polyps seen were thought to have caused the patient's symptoms. Strictures obviously could lead to constipation, as well as diarrhea due to overflow, and therefore progressing to an alteration in bowel habits. Overall, our findings are still supportive and consistent with the ASGE guidelines, of moderate quality of evidence, for the role of endoscopy in constipation. That being, that GI endoscopy should not be performed in the initial evaluation of patients presenting with symptoms of chronic constipation in the absence of alarm features or suspicion of organic GI disease. ${ }^{1}$ Also, when comparing EGD/Colonoscopy together with Colonoscopy alone, the diagnostic yield was higher for anatomic findings. This was an expected conclusion from the study. It was unclear why, in three patients, EGD was performed multiple times.

As far as the histological diagnosis, a total of 32 patients (10.77\%) were found to have a definitive diagnosis leading to chronic $\mathrm{ABH}$. These diagnoses include inflammatory bowel disease, ischemia, microscopic colitis, infectious etiology, cancer, and celiac disease. There were 13 cases $(4.4 \%$ of the total population or even more interestingly, $40.63 \%$ of the 32 patients that had a definitive cause for chronic $\mathrm{ABH}$ ) of microscopic colitis. In other words, $40.63 \%$ of the 32 had a normal anatomical appearing endoscopy. This confirms the indication to perform biopsies for chronic $\mathrm{ABH}$, specifically chronic diarrhea. According to the ASGE guidelines, however low quality, on the role of endoscopy for diarrhea, ${ }^{2}$ colonoscopy with random 
biopsies of the right and left side of the colon and sigmoidoscopy as an alternative option should be performed for chronic unexplained diarrhea. With a moderate quality of evidence, the same guidelines recommend EGD with small bowel biopsy in patients with chronic diarrhea. A total of 3 patients ( $1 \%$ of the total patients or $3.37 \%$ of those 89 patients who had EGD or EGD/Colonoscopy performed) had a new histological diagnosis of Celiac disease, signifying a low yield of endoscopy in general, but a slightly higher yield when performing an EGD, for this specific diagnosis. This justifies the need to perform serology, which will raise the suspicion for Celiac disease prior to endoscopy.

There were many strengths to this study. Previous studies have reported on the yield of endoscopy for abdominal pain or constipation and constipation alone. These include "Appropriateness of Colonoscopy: Lower Abdominal Pain or Constipation" by V De Bosset et al. ${ }^{3}$ who discussed the diagnostic yield of GI endoscopy for abdominal pain or constipation, and not chronic constipation or altered bowel habits alone. Another study, "Constipation is Not Associated with an Increased Rate of Findings on Colonoscopy: Results from a National Endoscopy Consortium" by M Gupta et al. ${ }^{4}$ included patients with anemia, hematochezia, weight loss, and abdominal pain, aside from just constipation. Our study examined patients with chronic constipation without an indication of anemia or hematochezia, to name a few. With these studies in mind, the goal of our study was directed towards fewer indications for endoscopy, leading to less variability. Also, the majority of endoscopists (255 of the 297 or $85.86 \%$ ) were performed by gastroenterologist of the same private practice leading to more consistency in the endoscopy reports and endoscopy techniques. But, there was no standardization to the endoscopy reports, so several patients were excluded if the definition of chronic diarrhea or chronic constipation was not specified, making this study more selective for chronic diarrhea and constipation.

However, there were also several weaknesses to this study. In reference to the proper definition of chronic diarrhea and chronic constipation being $>6$ weeks, the proposed population size to achieve a confidence interval of $95 \%$ was calculated to be 504 ; however this study only included 297, giving it a higher margin for error. When excluding patients from the study, a thorough examination of proper workup prior to endoscopy was not able to be assessed since this was a retrospective chart review of open access endoscopy. Records from the physicians' office were not available, making it unclear that noninvasive testing (stool studies, serology, dietary modification, etc.) had been done. We used timeframe and history, rather than volume or weight, in the chart to define diarrhea and constipation, having left it up to the endoscopists to have verified that the patient indeed had chronic diarrhea or constipation.

\section{Conclusion}

In summary, we found our anatomic diagnostic yield of endoscopy to be $14.48 \%$ and histological diagnostic yield of endoscopy to be $10.77 \%$. This tells us, as we suspected, that the overall endoscopic yield is low, even considering the margin of error due to the small population size. Further studies should be done in a prospective manner with clearly defined indications and proper workup having already been performed prior to endoscopy. The question is raised as to what diagnostic yield is acceptable to undergo the risks associated with endoscopy, especially in the elderly population. In addition, costeffectiveness should also be evaluated further. ${ }^{5-8}$

\section{Acknowledgments}

None.

\section{Conflicts of interest}

The authors declare no conflict of interest.

\section{References}

1. De Bosset V, Gonvers JJ, Vader JP, et al. Appropriateness of colonoscopy: lower abdominal pain or constipation. Endoscopy. 1999;31(8):637-640.

2. Gupta M, Holub J, Knigge K, et al. Constipation is not associated with an increased rate of findings on colonoscopy: results from a national endoscopy consortium. Endoscopy. 2010;42(3):208-212.

3. Eisen GM, Dominitz JA, Faigel DO, et al. Use of endoscopy in diarrheal illnesses. American Society for Gastrointestinal Endoscopy. 2001;54(6):821-823.

4. Lasson A, Kilander A, Stotzer PO. Diagnostic yield of colonoscopy based on symptoms. Scandinavian Journal of Gastroenterology. 2008;43(3):356-362.

5. Shen B, Khan K, Ikenberry SO, et al. The role of endoscopy in the management of patients with diarrhea. Gastrointestinal Endoscopy. 2010;71(6):887-892.

6. Cash BD, Acosta RD, Chandrasekhara V, et al. The role of endoscopy in the management of constipation. Gastrointestinal Endoscopy. 2014;80(4):563-565.

7. Qayed Emad, Shanthi Srinivasan, Nikrad Shahnavaz. Sleisenger and Fordtran's Gastrointestinal and Liver Disease. (10th edn), Elsevier, USA, 2017;183.

8. Wasson JH, Sox HC, Sox CH. The diagnosis of abdominal pain in ambulatory male patients. Medical Decision Makin. 1981;1(3):215-224. 